\title{
Traveling Front Solutions to Directed Diffusion Limited Aggregation, Digital Search Trees and the Lempel-Ziv Data Compression Algorithm
}

\author{
Satya N. Majumdar \\ Laboratoire de Physique Théorique (FER 2603 du CNRS), Université Paul Sabatier, 31062 Toulouse Cedex, France
}

\begin{abstract}
We use the traveling front approach to derive exact asymptotic results for the statistics of the number of particles in a class of directed diffusion limited aggregation models on a Cayley tree. We point out that some aspects of these models are closely connected to two different problems in computer science, namely the digital search tree problem in data structures and the Lempel-Ziv algorithm for data compression. The statistics of the number of particles studied here is related to the statistics of height in digital search trees which, in turn, is related to the statistics of the length of the longest word formed by the Lempel-Ziv algorithm. Implications of our results to these computer science problems are pointed out.
\end{abstract}

PACS numbers: 02.50.-r, 89.75.Hc, 89.20.Ff

\section{INTRODUCTION}

The simple model of diffusion limited aggregation (DLA), ever since its introduction by Witten and Sander in 1981 [1], has continued to play a central role in understanding the fractal growth phenomena. Besides raising a number of conceptual issues regarding pattern formation, this model has also found numerous applications in physical processes ranging from dielectric breakdown [2] and Hele-Shaw fluid flow [3] to electrodeposition [4] and dendritic growth [5]. In the simplest version of this model, one considers, for example, a square lattice where the origin is a seed. Particles are released sequentially from the boundary. Each particle performs a random walk in space and when it comes in contact with the growing cluster around the central seed, it sticks to the cluster and thus the cluster grows. This growing DLA cluster has a fractal structure with many branches that are separated by deep 'fjords'. Despite various advances, characterizing this fractal pattern quantitatively has remained a major theoretical challenge for the past two decades [6]. One clear picture that has emerged out of various studies is that the key effect responsible for this complex pattern is the dynamical 'screening' : a newly arriving particle has more probability to attach to the 'tip' sites compared to other boundary sites that are deep inside a 'fjord'. As a result, the faster growing parts of the cluster boundary shield or screen other boundary sites from further growth.

To understand this dynamical screening effect more quantitatively, it is desirable to construct a simpler model which incorpoartes the screening effect and yet is analytically tractable. Bradley and Strenski [7] introduced such a model where particles undergo a directed diffusion limited aggregation (DDLA) on a Cayley tree. Physically this corresponds to the situation when there is a strong external field such as the gravity or an electric field which forces the particles to choose an overall direction of mo- tion. In this DDLA model, one starts with a Cayley tree of height $l$ (see Fig. 1) where all the $2^{l}-1$ sites of the lattice are initially empty. Then a particle is introduced at the top site and it performs a directed (downwards) random walk (from any site it choooses one of the two daughter sites at random and moves there) till it reaches one of the bottom leaves and can descend no more. It then occupies that leaf site.

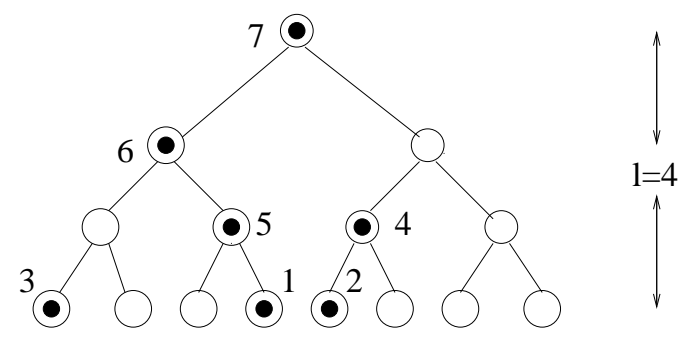

FIG. 1. A typical history of the DDLA process till saturation on a Cayley tree with height $l=4$. The occupied nodes contain a black filled circle inside them and the number next to an occupied site denotes the particle number.

Then a second particle is introduced from the top site and it also performs a directed random walk. This second particle will stop if it happens to reach a site such that atleast one of the daughter nodes of that site is already occupied. It can not descend any more and it rests at that site for all subsequent times. Note that in this model, each site can contain at most one particle. Then a third particle is released from the top and so on. Basically, after reaching any site, say the $i$-th site, a particle 
attempts to hop down to one of the two daughter nodes of $i$ and it actually moves to the target site provided both the daughter nodes of $i$ are empty. If atleast one of them is occupied, the particle can not descend any further and it rests at site $i$ forever. Then the next particle is added and the process continues until no more particles can be put in, i.e. when the top site gets occupied. The tree is then said to be saturated. One such history of the process till its saturation is shown in Fig. 1. A typical snapshot of the saturated tree (see Fig. 1) shows that the cluster has voids of various sizes. A useful quantity to characterize the pattern of the cluster is the total number of particles $n_{l}$ in the saturated tree. Clearly $n_{l}$ is a random variable, varying from one history of the process to another. The quantities of central importance in this problem are the average density, $\rho_{l}=\left\langle n_{l}\right\rangle /\left[2^{l}-1\right]$ and the fluctuations of $n_{l}$ around its average value. How do these two quantities behave asymptotically for a large tree, i.e. when $l \rightarrow \infty$ ?

While it was easy to write down the basic recursion relations (see later in Section II) for certain probabilities associated with the DDLA process on a tree, they turned out to be nonlinear [7] and hence it was difficult to determine even the asymptotic behavior of $\rho_{l}$ for large l. Bradley and Strenski studied the recursion relations numerically and found, somewhat unexpectedly, that $\rho_{l}$ decays slower than exponentially with $l$ for large $l$ but the precise nature of this decay was not evident from their numerics [7]. Later Aldous and Shields [8] studied via rigorous probabilistic methods a completely different model namely a continuous time version of the so called digital search tree (DST) problem relevant in computer science $[9,10,8,11-14]$. As we will see later in Section-VI, these two models namely the DDLA and the DST share the same recursion relation, though for very different quantities. The rigorous results of Aldous and Shields [8], when translated back in the DDLA language, would indicate a streched exponential decay for the average density, $\rho_{l} \sim 2^{-\sqrt{2 l}}$ for large $l$. Recently a more refined result on DST was derived by Knessl and Szpankowsky [15]. Unaware of the DDLA or the DST model, Hastings and Halsey also studied independently a related model recently [16] and used extremal arguments to conclude the same streched exponential decay for the average density.

The methods used by the mathematicians, though rigorous, lack physical transparency. On the other hand, the extremal arguments used by Hastings and Halsey, though physically intuituive, are heuristic. Moreover, it is not easy to derive quantitative results regarding fluctuations of the number of particles $n_{l}$ via these methods. For example, how does the width $w(l)=\sqrt{\left\langle n_{l}^{2}\right\rangle-\left\langle n_{l}\right\rangle^{2}}$ behave as a function of $l$ ? Besides, none of these methods are easily adaptable to extract the leading asymptotic behaviors in more general models such as one that we will consider in this paper. Our approach in this paper would be to use the powerful techniques (suitably adapted for our problem) of traveling fronts, originally developed in the context of nonlinear reaction-diffusion systems and population dynamics [17-19]. The techniques of traveling fronts have found a host of very useful applications [20]. Recently we have pointed out that in many extreme value problems in both physics and computer science, one can successfully use the traveling front techniques to derive exact asymptotic results for the statistics of the extremum of a set of correlated random variables [21-24]. The present paper points out yet another useful application of the traveling front techniques, namely in a generalized DDLA problem with relevance to a class of search tree problems in computer science.

The traveling front method, though technically not rigorous in the strict mathematical sense, has the following advantages over the other methods used in the DDLA problem: (i) this method is not model specific, is more general and is easily adapdable to more general models such as the ones that will be studied in this paper, (ii) it is easy to implement and is physically transparent and (iii) it provides a very cheap way to extract the leading asympotic behavior exactly without using too much mathematics. Besides rederiving the known results in the standard DDLA problem, this method also allows us to derive many new results in more generalized models. For example, we show that in the DDLA model, the random variable $n_{l}$ approaches to its average value, $n \rightarrow\left\langle n_{l}\right\rangle$ in the $l \rightarrow \infty$ limit. This is the example of the extreme concentration of measure, i.e., the distribution of $n_{l}$ tends to a delta function. In particular, we show that the width of the distribution decays slowly as a power law, $w(l) \sim l^{-1 / 2}$ as $l \rightarrow \infty$. Furthermore, we point out the close connection between the DDLA problem and the DST problem widely studied by computer scientists $[9,10,8]$. The later problem is also related to the well known Lempel-Ziv algorithm used in data compression [25]. Some of the results derived in this paper will constitute new results in these computer science problems.

The rest of the paper is organized as follows. In the next section we introduce a generalized b-DDLA model (where $b$ is a positive integer). The original DDLA model of Bradley and Strenski is a special case of this b-DDLA model with $b=1$. We then derive the asymptotic statistics of the number of particles in the b-DDLA model using the traveling front technique, suitably adapted for this model. In Section III, we generalize these results to the case when the Cayley tree has $m>2$ branches. Section IV considers the DDLA model with a bias when a particle has more probability to go to the left branch compared to the right one. In Section $\mathrm{V}$, we point out the detailed connections between the DDLA model, the DST problem in computer science, and the Lempel-Ziv parsing algorithm and discuss the implications of our re- 
sults for the generalized b-DDLA model in the context of computer science. Finally a brief summary and a conclusion along with a list of open problems are offered in Section VI.

\section{THE B-DDLA MODEL AND ITS TRAVELING FRONT SOLUTION}

Here we introduce a generalized b-DDLA model where the 'hard' screening of the usual DDLA model is 'softened' in the following sense. As in the usual DDLA model, one starts with an empty Cayley tree of height $l$ and the particles are introduced sequentially at the top site and they go down the tree one at a time by performing a random walk. However, now each site can contain at most $b$ particles where $b$ is a positive integer. During its journey downward, when a new particle arrives at an empty site, say the $i$-th site, it tries to move to one of the daughter nodes of $i$ chosen at random. It actually moves to the target site provided both the daughter sites contain less than $b$ particles. If either of them contains $b$ particles, i.e. is completely full, then the incoming particle can not move down any further and it then stays at site $i$ forever. Thus, in this model, a site can incorporate 'screening' if and only if it has full capacity, i.e. when it has $b$ particles. Otherwise it fails to screen. This model thus mimics the physical situation when one single particle is incapable of stopping an incoming particle to go down, but the screening comes into play only as a collective effect when there are $b$ particles in the site. This is like a tunnelling effect, where a particle can go through a barrier provided the barrier is not too high. However, the rate of tunnelling goes to zero when the barrier height crosses a threshold. In our model, the parameter $b$ acts like the threshold value. Clearly, for $b=1$ this model reduces to the original DDLA model studied by Bradley and Strenski [7].

As in the $b=1$ case, the tree is going to be saturated after a finite number of particles have been added to it. This happens when the top site contains $b$ particles. No further particles can then be put in. The number of particles $n_{l}$ required to saturate the tree of height $l$ is clearly a random variable, fluctuating from one history of the process to another. The main question we address is: what is the statistics of $n_{l}$ for large $l$ ? In particular, we would compute the average density at saturation, $\rho_{l}=\left\langle n_{l}\right\rangle /\left[2^{l}-1\right]$ and the width of the distribution, $w(l)=\sqrt{\left\langle n_{l}^{2}\right\rangle-\left\langle n_{l}\right\rangle^{2}}$ for large $l$.

Following Bradley and Strenski [7] for the $b=1$ case, we define $G_{l}(n)$ to be the probability that the tree of height $l$ is not saturated after the addition of $n$ particles, i.e. the top site is not yet filled by $b$ particles after $n$ particles have been added to the tree. It is easy to see that $G_{l}(n)$ satisfies the following recursion relation,

$$
G_{l+1}(n+b)=\frac{1}{2^{n}} \sum_{n_{1}=0}^{n}\left(\begin{array}{c}
n \\
n_{1}
\end{array}\right) G_{l}\left(n_{1}\right) G_{l}\left(n-n_{1}\right)
$$

for all $l \geq 1$ and $n \geq 0$. It is useful to think of $l$ as 'space' and $n$ as 'time'. The Eq. (1) is supplemented with the 'boundary' condition, $G_{1}(n)=1$ for all $0 \leq n \leq(b-1)$ and $G_{1}(n)=0$ for all $n \geq b$ and the 'initial' condition, $G_{l}(n)=1$ for $0 \leq n \leq(b-1)$ for all $l \geq 1$. The recursion relation in Eq. (1) is easy to understand. Suppose we have added $(n+b)$ particles to a tree of height $(l+1)$ (the left hand side of Eq. (1)). The condition that the top site is not yet filled by $b$ particles indicates that before the addition of the last $b$ particles, the two daughter nodes of the top site must have both remained unsaturated. This is becuase, if either one or both of them had been saturated after the addition of $n$ particles, then any further added particle would not be able to go down and would rest at the top site, and hence the top site would then get saturated after the addition of $(n+b)$ particles. The two daughter nodes are the roots of two uncorrelated subtrees, each of height $l$. Hence the probability that both remain unsaturated is given by their product. Also, the number of particles $n_{1}$ that enter, for example, to the left subtree (out of a total number of $n$ particles that enter both subtrees) must have a binomial distribution, thus explaining the right hand side of Eq. (1).

Note that for fixed $n>0$, the probability $G_{l}(n) \rightarrow 0$ as $l \rightarrow 1$ and $G_{l}(n) \rightarrow 1$ as $l \rightarrow \infty$. For later analysis, it turns out to be convenient to define the complementary probability, $F_{l}(n)=1-G_{l}(n)$, that has the opposite behavior as a function of $l$, namely $F_{l}(n) \rightarrow 1$ as $l \rightarrow 1$ and $F_{l}(n) \rightarrow 0$ as $l \rightarrow \infty$ for any fixed $n>0$. The quantity $F_{l}(n)$ denotes the probability that the tree of height $l$ gets saturated before $n$ particles are added. From Eq. (1), one finds that $F_{l}(n)$ satisfies the recursion,

$$
\begin{aligned}
F_{l+1}(n+b) & =\frac{1}{2^{n-1}} \sum_{n_{1}=0}^{n}\left(\begin{array}{c}
n \\
n_{1}
\end{array}\right) F_{l}\left(n_{1}\right) \\
& -\frac{1}{2^{n}} \sum_{n_{1}=0}^{n}\left(\begin{array}{c}
n \\
n_{1}
\end{array}\right) F_{l}\left(n_{1}\right) F_{l}\left(n-n_{1}\right),
\end{aligned}
$$

with the boundary condition, $F_{1}(n)=0$ for $0 \leq n \leq$ $(b-1)$ and $F_{1}(n)=1$ for $n \geq b$ and the initial condition, $F_{l}(n)=0$ for $0 \leq n \leq(b-1)$ for all $l \geq 1$. It is useful to think by fixing the 'time' $n$ while varying the 'space' $l$. Clearly $F_{l}(n) \rightarrow 0$ as $l \rightarrow \infty$, since almost surely a tree of infinite height will not be saturated before the addition a fixed, finite number $n$ of particles. On the other side, for fixed $n, F_{l}(n) \rightarrow 1$ as $l \rightarrow 0$. For a given fixed $n$, as one increases $l$ from 0 to $\infty$, the function $F_{l}(n)$ starts off at the value 1 at $l=0$ and then drops off to 0 beyond some characteristic length scale $l^{*}(n)$, as shown schematically in Fig. (2). As $n$ increases, this characteristic length scale $l^{*}(n)$ also increases (see Fig. (2)), thus giving rise 
to a traveling front structure with the front located at $l^{*}(n)$. In fact, we will see later that in the limit of large $l$ (when one can treat $l$ as a contiuous variable) and large $n$, the width $w(n)$ of the front goes to zero, indicating that asymptotically the function $F_{l}(n)$ becomes a Heaviside theta function, $F_{l}(n) \rightarrow \theta\left(l^{*}(n)-l\right)$.

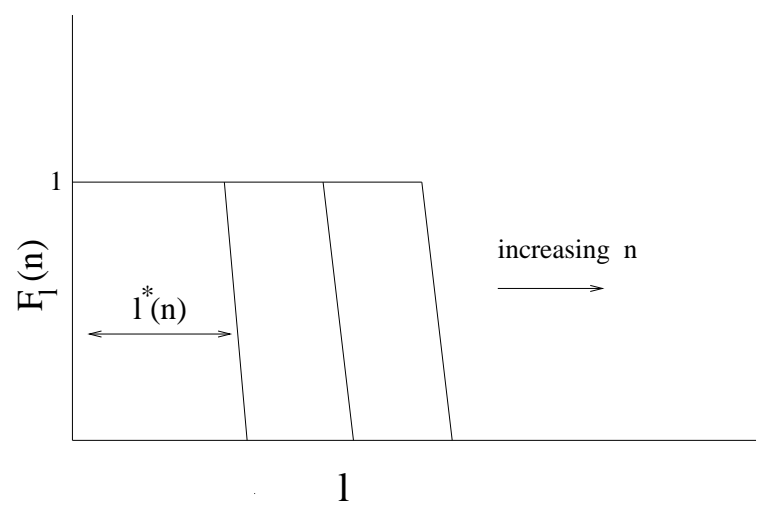

FIG. 2. Schematic behavior of the probability $F_{l}(n)$ as a function of $l$ for different fixed values of $n$. The three curves correspond to different values of $n$ increasing from left to right. The $y$ axis is dimensionless while the $x$ axis has arbitrary units.

Note that Eq. (2) is nonlinear and hence is diffcult to solve exactly. However, the exact asymptotic informations regarding the position $l^{*}(n)$ of the front and its width can be deduced by adapting the traveling front techniques that were originally devised to deal with nonlinear partial differential differential equations in reaction-difffusion systems [17] and pupulation dynamics $[18,19]$. The basic idea behind this approach is very simple. If there is a front $l^{*}(n)$, then ahead of the front $l>l^{*}(n), F_{l}(n)$ is very small and hence one can neglect the nonlinear term (the second term) on the right hand side of Eq. (2) and one simply gets a linear recursion,

$$
F_{l+1}(n+b) \approx \frac{1}{2^{n-1}} \sum_{n_{1}=0}^{n}\left(\begin{array}{c}
n \\
n_{1}
\end{array}\right) F_{l}\left(n_{1}\right) .
$$

Suppose one could solve this linear equation exactly satisfying the required initial condition. Now one expects that the solution of the linear equation (3) and the 'real' solution of the nonlinear equation (2) will coincide in the regime beyond the front, i,e. for $l>l^{*}(n)$. On the other hand, the two solutions will start differing from each other as one decreases $l$ below $l^{*}(n)$, where the solution of the nonlinear equation (2) will tend to 1 where as the solution of the linear equation (3) will grow beyond 1 with decreasing $l$ (as there is no nonlinear term to control the solution). Thus, as one decreases $l$ from infinity, the front position $l^{*}(n)$ is approximately the value of $l$ at which the solution of the linear equation becomes $\sim O(1)$. Thus, according to this approach, one first solves the linear equation $(3)$ to obtain $\left.F_{l}(n)\right|_{\text {linear }}$ and then reads off the front position $l^{*}(n)$ from the condition, $\left.F_{l^{*}(n)}(n)\right|_{\text {linear }} \approx O(1)$. By $O(1)$, one means that at $l=l^{*}(n)$, the solution $F_{l}(n)$ should not diverge or decay exponentially with increasing $n$. Note that this is a slightly generalized version of the usual traveling front method [20,21] where one usually has a linear operator with constant coefficients which admits an exponentially decaying solution of the form $\exp [-\lambda(x-v t)]$ with constant width. The present approach is more general and works even when the linear operator does not admit an exponentially decaying solution with a constant width.

Under this traveling front approach, our task thus reduces to solving the linear equation (3) which, however, is still nontrivial. To proceed, we define a somewhat unusual generating function,

$$
\tilde{F}_{l}(s)=\sum_{n=0}^{\infty} F_{l}(n) \frac{1}{(1+s)^{n+1}}
$$

Using Eq. (3) one can then show that $\tilde{F}_{l}(s)$ satisfies a rather simple recursion in $l$,

$$
\tilde{F}_{l+1}(s)=\frac{4}{(1+s)^{b}} \tilde{F}_{l}(2 s)
$$

for all $l \geq 1$. The steps leading to Eq. (5), starting from Eqs. (3) and (4), are not completely straightforward. Hence we present this derivation in the Appendix. The recursion in Eq. (5) starts with the initial value $\tilde{F}_{1}(s)$ which needs to be calculated separately. Noting that $F_{1}(n)=0$ for $0 \leq n \leq(b-1)$ and $F_{1}(n)=1$ for $n \geq b$, we find from the definition in Eq. (4), $\tilde{F}_{1}(s)=1 /\left[s(1+s)^{b}\right]$. Iterating Eq. (5) and using the expression for $l=1$, we get

$$
\begin{aligned}
\tilde{F}_{l}(s) & =\frac{2^{l-1}}{s\left[(1+s)(1+2 s) \ldots\left(1+2^{l-1} s\right)\right]^{b}} \\
& =\frac{2^{l-1}}{s} \exp \left[-b \sum_{k=0}^{l-1} \ln \left(1+2^{k} s\right)\right],
\end{aligned}
$$

for all $l \geq 1$.

We then write the sum inside the exponential in Eq. (6) in two parts using the Euler-Maclaurin summation formula, $S(l, s)=\sum_{k=0}^{l-1} \ln \left(1+2^{k} s\right)=I(l, s)+R(l, s)$ where $I(l, s)=\int_{0}^{l} \ln \left(1+2^{x} s\right) d x$ and $R(l, s)=S(l, s)-$ $I(l, s)$ is the residual term. The integral $I(l, s)$ can be done and one gets, $I(l, s)=\left[Y\left(2^{l} s\right)-Y(s)\right] / \ln 2$ where the function $Y(z)$ is given by

$$
Y(z)= \begin{cases}\sum_{n \overline{\overline{2}}}^{\infty}(-1)^{n-1} z^{n-1} n^{-2} & \text { for } z \leq 1 \\ \frac{1}{2} \ln ^{2} z-\ln (1+1 / z)+\ln 2+\pi^{2} / 12 & \text { for } z>1\end{cases}
$$


The structure of these expressions suggests a natural scaling limit, $s \rightarrow 0, l \rightarrow \infty$ but keeping the product $z=s 2^{l}$ fixed but arbitrary. We also treat $l$ as a continuous variable in this limit. Furthermore, we focus only near the tail of the scaling regime, i.e. when $z>>1$. In this regime, it is sufficient to keep only the first term in the second line of Eq. (7) for the expression of $Y(z)$. Besides, one can also check that the residual term is subleading in this regime. Keeping only the leading terms we get

$$
\tilde{F}_{l}(s) \approx \frac{1}{s} \exp \left[\ln 2\left(l-\frac{b}{2}\left(l+\log _{2}(s)\right)^{2}\right)\right] .
$$

We still need to invert the generating function in Eq. (4) to obtain the asymptotic behavior of $F_{l}(n)$. The scaling limit corresponds to taking $n \rightarrow \infty, l \rightarrow \infty$ but keeping the ratio $2^{l} / n$ fixed but arbitrary. Using Eq. (8) and inverting Eq. (4) (using the Bromwich inversion formula and then using the standard steepest descent method), we find the following leading asymptotic behavior,

$$
F_{l}(n) \approx \exp \left[\ln 2\left(l-\frac{b}{2}\left(l-\log _{2}(n)\right)^{2}\right)\right],
$$

valid in the tail $l>>\log _{2}(n)$.

Having obtained the asymptotic solution (9) of the linear equation (3), the location of the front $l^{*}(n)$ can be read off from the equation, $\left.F_{l^{*}}(n)\right|_{\text {linear }} \sim O(1)$. Using this criterion in Eq. (9), we find that the front position $l^{*}(n)$ is given by the quadratic equation,

$$
l^{*}-\frac{b}{2}\left[l^{*}-\log _{2}(n)\right]^{2}=0 .
$$

As we decrease $l$ from $\infty$, we will encounter the larger root first, which will correctly locate the front position. From Eq. (10), we get, for large $n$, the asymptotic front position

$$
l^{*}(n) \approx \log _{2}(n)+\sqrt{\frac{2}{b} \log _{2}(n)} .
$$

Furthermore, substituing $l=l^{*}(n)+y$ in Eq. (9) and expanding for small $y$, we find to leading order, $F_{l}(n) \sim \exp \left[-\sqrt{2 b \ln (2) \ln (n)}\left(l-l^{*}(n)\right)\right]$, indicating that the width of the distribution, characterizing the fluctuation of $l$ around its average value $l^{*}(n)$, decreases extremely slowly with $n$ as,

$$
w(n) \approx 1 / \sqrt{2 b \ln (2) \ln (n)},
$$

as $n \rightarrow \infty$. The fact that the width vanishes in the $n \rightarrow$ $\infty$ limit shows that the probability $F_{l}(n) \rightarrow \theta\left(l^{*}(n)-l\right)$, thus indicating an extreme concentration of measure, i.e. the random variable $l \rightarrow l^{*}(n)$.

In the above analysis, we kept $n$ fixed and studied the behavior of $F_{l}(n)$ as a function of $l$. Alternately, as is more suited for the DDLA problem, one can keep $l$ fixed and vary $n$. It follows from Eq. (9) that in the scaling limit mentioned above, the random variable $\log _{2}(n)$ approaches to its mean value $\left\langle\log _{2}(n)\right\rangle=l-\sqrt{2 l / b}$. Due to the extreme concentration of measure, it follows then that $n \rightarrow\left\langle n_{l}\right\rangle \approx 2^{l-\sqrt{2 l / b}}$ in the scaling limit. This means that the average density $\rho_{l}=\langle n\rangle /\left(2^{l}-1\right)$ decays as a stretched exponential for large $l$,

$$
\rho(l) \approx 2^{-\sqrt{2 l / b}} .
$$

Besides, substituting $\log _{2}(n)=\left\langle\log _{2}(n)\right\rangle-y_{1}$ in Eq. (9) and expanding for small $y_{1}$, we find, $F_{l}(n) \approx$ $\exp \left[-\ln (2) \sqrt{2 b l} y_{1}\right]$. This indicates that as a function of $l$, the width of the random variable $\log _{2}(n)$ around its average value $\left\langle\log _{2}(n)\right\rangle=l-\sqrt{2 l / b}$ decreases algebraically for large $l$

$$
w(l) \approx \frac{1}{\sqrt{2 b \ln ^{2}(2) l}} .
$$

Note that the leading order behaviors of the widths in Eqs. (12) and (14) are compatible with each other with the identification $n \approx 2^{l}$. The Eqs. (11), (12), (13) and (14) constitute the main results of this section.

\section{GENERALIZATION TO A TREE WITH $M$ BRANCHES}

In this section we generalize our results for the bDDLA in the previous section (obtained for a tree with $m=2$ branches) to a tree with $m \geq 2$ branches. In this case, during its downward journey from the top, a particle from a given site attempts to hop to any of the $m$ daughter nodes with equal probability $1 / m$ and can actually hop to the target site provided none of the $m$ daughter nodes is full with $b$ particles. If it fails to hop, the particle stays at its current site for all subsequent time. The probability $G_{l}(n)$ that a tree of height $l$ is yet to be saturated after the addition of $n$ particles satisfies the generalized recursion relation,

$$
G_{l+1}(n+b)=\frac{n !}{m^{n}} \sum_{n_{i}=0}^{m} \prod_{i=1}^{m} \frac{G_{l}\left(n_{i}\right)}{n_{i} !}
$$

where the variables $n_{i}$ 's satisfy the constraint $\sum_{i=1}^{m} n_{i}=$ $m$. Thus the binomial coefficient in Eq. (1) of the previous section gets replaced by a multinominal. The rest of the analysis is straightforward and similar to the previous section. We define as usual, the complementary probability, $F_{l}(n)=1-G_{l}(n)$, which satisfies the recursion,

$$
\begin{aligned}
F_{l+1}(n+b) & =\frac{1}{m^{n-1}} \sum_{n_{i}=0}^{m} \frac{n !}{\prod_{i=1}^{m} n_{i} !} F_{l}\left(n_{1}\right) \\
& + \text { nonlinear terms, }
\end{aligned}
$$


where we have used the symmetry that all branches are similar to each other.

As before, we solve the equation (16) retaining only the linear terms and neglecting the nonlinear terms. We define the generating function as in Eq. (4). Following the derivation presented in the Appendix and using the initial condition, we get the solution

$$
\tilde{F}_{l}(s)=\frac{m^{l-1}}{s\left[(1+s)(1+m s) \ldots\left(1+m^{l-1} s\right)\right]^{b}} .
$$

The asymptotic analysis is exactly similar to the previous section, except that the proper scaling limit now is $s \rightarrow 0, l \rightarrow \infty$ but keeping the product $s m^{l}$ fixed but arbitrary. We do not repeat the steps here but present only the final results. We find that as in the $m=2$ case, there is a front whose asymptotic location $l^{*}(n)$ is given by

$$
l^{*}(n) \approx \log _{m}(n)+\sqrt{\frac{2}{b} \log _{m}(n)},
$$

and in the limit $n \rightarrow \infty$, the width $w(n)$ of the front vanishes slowly as

$$
w(n) \approx 1 / \sqrt{2 b \ln (m) \ln (n)} .
$$

Similarly, we find that for fixed but large $l$, the average density varies as a strectched exponential,

$$
\rho(l) \approx m^{-\sqrt{2 l / b}}
$$

and the width $w(l)$ of the random variable $\log _{m}(n)$ around its average value $\left\langle\log _{m}(n)\right\rangle=l-\sqrt{2 l / b}$ decreases algebraically for large $l$,

$$
w(l) \approx \frac{1}{\sqrt{2 b \ln ^{2}(m) l}} .
$$

Note that, interestingly, the asymptotic average value $\left\langle\log _{m}(n)\right\rangle=l-\sqrt{2 l / b}$ is actually independent of $m$.

\section{B-DDLA MODEL WITH BIASED HOPPING}

In this section we consider the b-DDLA model on a $m=2$ tree where the particles perform biased random walk on their way down the tree. More precisely, when a particle arrives at any given site $i$ on its way down after being introduced at the top site, it attempts to hop to the left daughter of the node $i$ with probability $p$ and to the right daughter with probability $q=1-p$. As before, it actually moves to the target site provided both the daughter nodes have less than $b$ particles. If at least one of them is full with $b$ particles, then the particle rests at site $i$ for all subsequent times. Then a new particle is added and the process continues till the top site gets filled with $b$ particles. Once again, we are interested in the statistics of the number of particles $n_{l}$ when the tree of height $l$ gets saturated. We define, as before, $G_{l}(n)$ to be the probability that the tree of height $l$ remains unsaturated, i.e. the top site remains unfilled upto the addition of $n$ particles. Following the same logic as in Section II, one easily finds the recursion relation,

$$
G_{l+1}(n+b)=\sum_{n_{1}=0}^{n}\left(\begin{array}{c}
n \\
n_{1}
\end{array}\right) p^{n_{1}} q^{n-n_{1}} G_{l}\left(n_{1}\right) G_{l}\left(n-n_{1}\right)
$$

for all $l \geq 1$ and $n \geq 0$.

The complementary probability, $F_{l}(n)=1-G_{l}(n)$, then satisfies the recursion,

$$
\begin{aligned}
F_{l+1}(n+b) & =\sum_{n_{1}=0}^{n}\left(\begin{array}{c}
n \\
n_{1}
\end{array}\right) p^{n_{1}} q^{1-n_{1}}\left[F_{l}\left(n_{1}\right)+F_{l}\left(n-n_{1}\right)\right] \\
& + \text { nonlinear terms, }
\end{aligned}
$$

with the boundary condition $F_{1}(n)=0$ for $0 \leq n \leq$ $(b-1)$ and $F_{1}(n)=1$ for $n \geq b$. As before, we solve the equation (23) keeping only the linear terms and neglecting the nonlinear terms. This is done via defining the generating function $\tilde{F}_{l}(s)$ as in Eq. (4). Following the same line of derivation presented in the Appendix, we obtain the following recursion relation,

$$
\tilde{F}_{l+1}(s)=\frac{1}{p(1+s)^{b}} \tilde{F}_{l}(s / p)+\frac{1}{q(1+s)^{b}} \tilde{F}_{l}(s / q),
$$

which starts from the initial function, $\tilde{F}_{1}(s)=1 /[s(1+$ $s)$. One can, in principle, iterate Eq. (24) starting with $l=1$ and obtain the expressions for $\tilde{F}_{l}(s)$ for all $l$. Fortunately, in the scaling regime $s \rightarrow 0$, one does not need the full expression for $\tilde{F}_{l}(s)$. Note that in the unbiased case $p=q=1 / 2$, the appropriate scaling regime was $s \rightarrow 0, l \rightarrow \infty$ but keeping the product $z=s 2^{l}$ fixed but arbitrary. In the biased case, it is clear from Eq. (24) that the appropriate scaling regime will be set by taking $s \rightarrow 0, l \rightarrow \infty$ but keeping the product $z=s \sigma^{l}$ fixed where $\sigma=\min (1 / p, 1 / q)$. Thus in this scaling limit, one can approximate Eq. (24) by

$$
\tilde{F}_{l+1}(s) \approx \frac{\sigma}{(1+s)^{b}} \tilde{F}_{l}(\sigma s) .
$$

The terms neglected in going to Eq. (25) from Eq. (24) only contribute to subleading order. Iterating the reduced Eq. (25) starting with $\tilde{F}_{1}(s)=1 /[s(1+s)]$, one obtains

$$
\tilde{F}_{l}(s) \approx \frac{1}{s\left[(1+s)(1+\sigma s)\left(1+\sigma^{2} s\right) \ldots\left(1+\sigma^{l-1} s\right)\right]^{b}} .
$$


We then invert the transform in Eq. (26) using the same asymptotic method as in Section II, the details of which we do not repeat. The final asymptotic form of the distribution $F_{l}(n)$ is given by,

$$
F_{l}(n) \approx \exp \left[-\frac{\ln (\sigma)}{2}\left(l-\log _{\sigma}(n)\right)^{2}\right],
$$

valid in the scaling regime, $n \rightarrow \infty, l \rightarrow \infty$ but with the ratio $y=2^{l} / n$ fixed at a large value $y>>1$. The front position can be read off from the condition $\left.F_{l^{*}}(n)\right|_{\text {linear }} \sim$ $O(1)$ which gives, to leading order, $l^{*}(n) \approx \log _{\sigma}(n)$. Note the difference with the unbiased case. Unlike the unbiased case in Section II where the width vanishes for large $n$, here the width of the distribution remains of $O(1)$ in the large $n \rightarrow \infty$ limit, $w(l) \rightarrow 1 / \sqrt{\ln \sigma}$. This result also indicates that the average density of particles varies as $\left\langle n_{l}\right\rangle \approx \sigma^{l}$ for large $l$. Thus, unlike the unbiased case where the average density decays as a stretched exponential for large $l$, the average density in the biased case decays exponentially for large $l$,

$$
\rho(l) \approx(\sigma / 2)^{l},
$$

where $\sigma=\min (1 / p, 1 / q)$. Besides, it follows from Eq. (27) that the fluctuations of the variable $\log _{\sigma}(n)$ around its average value $l$ are characterized by a Gaussian tail with width of $O(1)$.

\section{CONNECTION TO DIGITAL SEARCH TREES AND THE LEMPEL-ZIV PARSING ALGORTHIM}

In this section we point out the connection between our generalized b-DDLA model to the so called digital search tree problem in computer science $[9,10,8,11-13,26]$ which, in turn, is also related [14] to the Lempel-Ziv data compression algorithm [25]. Suppose we have a data string $\left\{x_{1}, x_{2}, \ldots, x_{n}\right\}$ which needs to be stored on a binary tree. According to the digital search tree (DST) algorithm, one proceeds as follows. Initially all the nodes of the tree are empty. The first arriving element $x_{1}$ is put at the root of the tree. Each node can contain at most one element. The second element $x_{2}$ is put at one of the daughter nodes of the root chosen at random. Then for the next element $x_{3}$, one again starts at the root and choses one of the daughter nodes at random. If the chosen node is empty, $x_{3}$ goes there. But if the chosen node, say $i$, happens to be the one that contains $x_{2}$, then one chooses one of the two daughter nodes of $i$ at random and puts $x_{3}$ there. Then one stores the fourth element $x_{4}$ following the same algorithm and so on. Essentially each element $x_{i}$ performs a directed random walk down the tree till it finds an empty site which it then occupies. The process stops when all the $n$ elements have been stored and the resulting tree is called a DST (see Fig. 3). Note that according to this DST algorithm, the actual value of a data element say $x_{i}$ is not important. This is contrast to other search trees, such as the random binary search trees $[9,10]$ where the actual value of $x_{i}$ is used in constructing the tree.

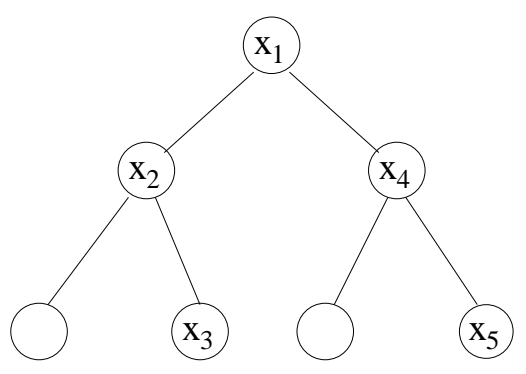

FIG. 3. A typical digital search tree constructed from a data string $\left\{x_{1}, x_{2}, x_{3}, x_{4}, x_{5}\right\}$ of 5 elements.

The statistics of various quantities such as the distribution of the number of occupied nodes at a given depth (known as the profile of the DST) have been studied in great detail in the computer science literature [14]. Flajolet and Richmond [12] introduced a generalized version of the DST where each node can contain at most $b$ elements. In this b-DST, an incoming element $x_{i}$ performs, as in the $b=1$ case, a directed random walk downwards. However, when $x_{i}$ reaches a new site, say $k$, it will stay forever at $k$ provided the number of already existing elements at $k$ is less than $b$. If the site $k$ already contains $b$ elements, then one chooses one of the daughter nodes of $k$ and the element $x_{i}$ hops there. This generalized b-DST problem has many applications in computer science, notably in the maintenance of paged hashing tables [12]. Flajolet and Richmond studied, for example, the average number of non-empty nodes in a b-DST as a function of the data size $n$ and the parameter $b$.

One important characteristic of a b-DST is its height. The height $l$ of a tree with $n$ elements is defined as the depth, counted from the root, of the farthest element in the tree. Clearly $l$ is a random variable, fluctuating from one realization of the tree to another and also it is an extreme variable (denoting the maximum depth). A natural question is: what is the probability distribution of the height? Let us define $Q_{l}(n)$ to be the probability that the height of tree with $n$ elements is $\leq l$. It is easy to see that $Q_{l}(n)$ satisfies the following recursion relation

$$
Q_{l+1}(n+b)=\frac{1}{2^{n}} \sum_{n_{1}=0}^{n}\left(\begin{array}{c}
n \\
n_{1}
\end{array}\right) Q_{l}\left(n_{1}\right) Q_{l}\left(n-n_{1}\right),
$$


for all $l \geq 1$ and $n \geq 0$, with the additional condition that $Q_{1}(n)=1$ for all $0 \leq n \leq b$ and $Q_{1}(n)=0$. For the case $b=1$, this recursion relation was recently studied by Knessl and Szpankowsky [15] using rigorous methods. The recurrence in Eq. (29) is a generalized version of the $b=1$ case and can be understood as follows. Consider a tree with a total number of $(n+b)$ elements. The first $b$ elements will be stored in the root and the rest of the $n$ elements will be distributed to the left and right daughter subtrees. The probability that one of the subtrees, say the left one, gets $n_{1}$ elements out of a total $n$ elements is simply given by the binomial distribution. Also, since the condition that the height of the full tree is $\leq l+1$ (the left hand side of Eq. (29)) indicates that the height of both of the daughter subtrees must be $\leq l$. Since the two daughter subtrees are completely independent, this probability is given by their product.

Note that the recursion relation in Eq. (29) for the height distribution $Q_{l}(n)$ in the b-DST is identical to the recursion in Eq. (1) in Section II for the probabilities $G_{l}(n)$ in the b-DDLA problem, except for the slight difference in the initial values $Q_{1}(n)$ and $G_{1}(n)$. This slight difference does not affect the asymptotic behaviors. So, one can apply all the results obtained via the traveling front approach in Section II for the b-DDLA model directly to the b-DST problem. In particular, the result in Eq. (11) indicates that the average height of the b-DST has the asymptotic following behavior for large $n$,

$$
l^{*}(n) \approx \log _{2}(n)+\sqrt{\frac{2}{b} \log _{2}(n)} .
$$

For $b=1$, this result coincides with that of Aldous and Shields [8] obtained by probabilistic methods. Note that for $b=1$ case, a more refined result including additional subleading terms to Eq. (30) was recently obtained in Ref. [15] using rigorous methods. However, for general $b$, we are not aware of any rigorous results in the computer science literature and our Eq. (30) seems to be the first result for the average height of a b-DST. Furthermore, Eq. (12) in Section II predicts that the standard deviation of the height around its average value decays extremely slowly with large $n, w(n) \approx 1 / \sqrt{2 b \ln (2) \ln (n)}$. This result on the variance of the height in b-DST also seems not to have been obtained by other methods before.

We now turn to the Lempel-Ziv algorithm for data compression [25]. The connection between this algorithm and the DST problem was known before $[8,14]$. The Lempel-Ziv algorithm is central to many universal data compression schemes and have many applications such as in the efficient transfer of data [14]. This basic scheme of this algorithm is very simple: it takes a given data string, say a sequence of binary digits such as 11000110111011110, and partitions it into 'words'. 'Words' are subsequences of variable sizes which are never repeated and are constructed by employing the rule that a new 'word' is the shortest subsequence not seen in the past as a 'word'. This is best understood by an example.

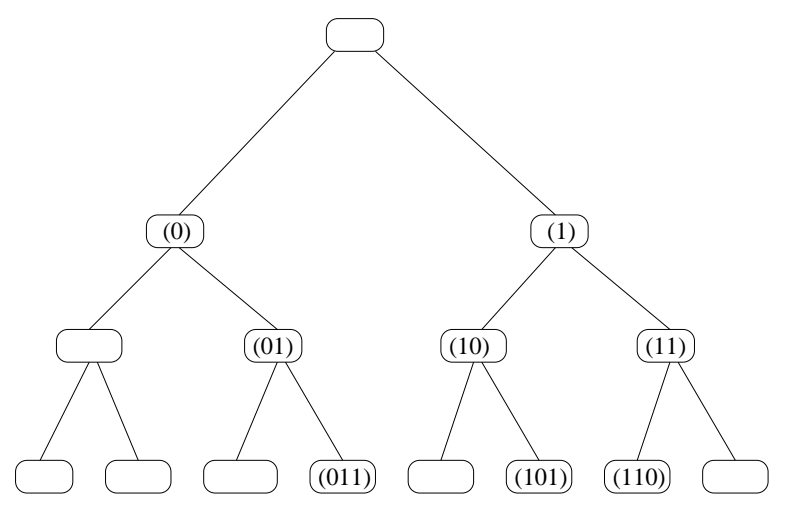

FIG. 4. The figure shows how the partitioning of a sequence 11000110111011110 into 'words' $(1)(10)(0)(01)(101)(11)(011)(110)$ using the Lempel-Ziv parsing algorithm can be represented as a digital search tree. The length of a 'word' is equal to its depth in the tree measured from the empty root at the top.

Consider the binary sequence 11000110111011110 and construct 'words' starting from the left end using the Lempel-Ziv algorithm. Starting from the left end, the first digit encountered is 1 . Since 1 has not occurred before as a 'word', one can form the first 'word' (1). Now we move to the next element which also happens to be 1 . But, now since (1) is already a 'word', the shortest segment we can use to form a 'word' is (10). Similarly, the next word would be $(0)$ since $(0)$ has not occurrred before as a 'word'. One keeps repeating the procedure and at the end, the original sequence is partitioned into the following sequence of 'words': $(1)(10)(0)(01)(101)(11)(011)(110)$. The original data is thus compressed into these words. Even though the 'words' are relatively short in the begining, it turns out that they become bigger quite rapidly. One of the interesting questions of practical importance in this scheme is the statistics of the longest 'word' when the original data string is random. For concreteness, let us consider a random binary sequence of initial data and focus on the first $n$ 'words'. Let $l$ be the length of the longest 'word' amongst these $n$ words. Clearly $l$ is a random variable since the underlying binary sequence is random. We are interested in the statistics of $l$ as a function of $n$.

There is a natural representation of this parsing algorithm in terms of a DST [14]. Consider a binary tree whose nodes are initially empty. In fact, the root of this tree is always going to be empty (see Fig. 4). Now we 
take the first of the Lempel-Ziv parsed 'words' and examine its first digit. If the first digit of this 'word' is 1 , we put this 'word' in the right daughter node of the root. On the other hand, if the first digit is 0 , we put this word at the left daughter node. This newly occupied node is now full and can not accomodate any other 'word'. Then we consider the second 'word' and look at its first digit. If the first digit is $1(0)$, we go to the right (left) daughter node. Let us call this node $i$. If this node $i$ is empty, we put the 'word' there. If $i$ is already occupied by the first 'word', then we need to examine the second digit of our second 'word' and depending on its value (1 or 0$)$, we go respectively to the right or to to the left daughter node of $i$ and put our second 'word' at this new site. This process is repeated until all the words are stored and the resulting tree is clearly a DST, since at each step the decision to go to the left or to the right occurs randomly (due to the randomness of the underlying binary sequence where each digit can be either 0 or 1 with equal probability). The construction of this DST from the parsed words $(1)(10)(0)(01)(101)(11)(011)(110)$ is shown in Fig. 4.

It is clear from the algorithm that the depth of a given 'word' in the DST (measured from the empty root) is precisely the equal to the length of the 'word' (see Fig. 4). In particular, the longest 'word' will also be the farthest from the root. Thus the length $l$ of the longest word is precisely the height of the corresponding DST. There is a generalized Lempel-Ziv algorithm where during the partitioning into 'words', any particular 'word' is allowed to be repeated at most $b$ times [14]. Then the corresponding DST is precisely a b-DST. Thus our results regarding the average height $l^{*}(n)$ and its width apply as well to the longest 'word' in the generalized Lempel-Ziv algorithm.

\section{CONCLUSIONS}

In this paper we have used a suitably adapted version of the traveling front approach to derive exact asymptotic results for the statistics of the number of particles in a generalized directed diffusion limited aggregation problem. We have pointed out a close connection of this problem to two separate problems in computer science, namely the digital search tree problem and the LempelZiv algorithm used for data compression. Our results for the number of particles in the generalized b-DDLA model have direct relevance to the statistics of height in the digital search tree problem and to the statistics of the longest word in the Lempel-Ziv algorithm.

The traveling front approach has recently been used successfully $[22,23]$ to derive exact asymptotic results for heights in a number of growing search tree problems in computer science. This paper shows that the scope of this approach can be extended to include yet another different class of search trees, namely the digital search tree.
The main advantage of this method is that it provides an easy way to derive the leading asymptotic behavior exactly in a variety of extreme value problems [21].

The present study leads to a number of interesting, open problems which we list below.

Undirected DLA problem on a tree: In this paper, we have focused on a directed model for simplicity, where the particles undergo diffusion but only in the overall downward direction. It would be interesting to extend the traveling front approach to an undirected model such as the one studied by Hastings and Halsey [16].

Subleading Corrections in the biased DDLA problem: Using traveling front approach, we managed to calculate only the leading behavior of the average density in the biased DDLA model (see Section IV). It would be interesting to compute the subleading corrections to this leading behavior.

DDLA model with stochastic screening: In this paper, we have studied a DDLA model where the screening is deterministic in the sense that a particle, on its way downwards, stops definitely when it reaches at a site such that at least one of the daughter nodes of that site is occupied. It would be interesting to consider a stochastic screening version defined as follows. For simplicity, we define the model for $b=1$ case, though it can be trivially generalized to $b>1$ case also. The particles are introduced sequentially from the top as before and a new particle is introduced only when the previous particle has completely stopped moving. On its way down, at each site $i$ a particle performs the following steps: if both the daughter nodes of $i$ are already occupied, the particle rests at $i$ for all subsequent times and then a new particle is added. If both the daughter nodes are empty, then the particle chooses one of the daughter nodes at random and moves there. If, however, only one of the daughter nodes (say the left one) is occupied but the other one (the right one) is empty, then with probability $p(0 \leq p \leq 1)$ the particle moves to the right daughter node and with probability $(1-p)$ it rests at $i$ for all subsequent times. Clearly the case $p=0$ corresponds to the deterministic screening model studied in Section II. On the other hand, for $p=1$, it is obvious that at the saturation the tree will be completely full and the density will be exactly 1 . It would be interesting to compute the statistics of density in this stochastic screening model.

Disordered b-DDLA model: In the present paper, we considered the b-DDLA model when all the nodes of the tree have the same capacity $b$. In a disordered version of the problem, this node capacity $b_{i}$ of a site $i$ may vary from one site to another. One can consider $b_{i}$ 's to be a set of quenched variables (as in the usual models in disorderd systems), each drawn independently from a specified distribution $p(b)$. Then, for a given fixed set of $b_{i}$ 's, one would first like to compute, for example, the average number of particles $\left\langle n_{l}\right\rangle$ at saturation, and then average this quantity over the disorder to obtain $\overline{\left\langle n_{l}\right\rangle}$, where $\ldots$ 
denotes the average over the $b_{i}$ 's. It would also be interesting to compute the sample to sample fluctuations of the average density $\left\langle n_{l}\right\rangle$.

\section{ACKNOWLEDGEMENTS}

I thank A.J. Bray, D.S. Dean, D. Dhar and P.L. Krapivsky for discussions. I thank P.L. Krapivsky also for pointing out the reference [7]. The hospitality of the Tata Institute, Bombay, where part of this work was done during a visit, is gratefully acknowledged.

\section{APPENDIX: DERIVATION OF THE GENERATING FUNCTION}

In this appendix, we present the derivation of Eq. (5) where $\tilde{F}_{l}(s)$ is defined in Eq. (4). Our starting point is the linear equation (3). We first define the exponential generating function,

$$
H_{l}(z)=\sum_{n=0}^{\infty} F_{l}(n) \frac{z^{n}}{n !} .
$$

Multiplying both sides of Eq. (3) by $z^{n} / n$ ! and summing over $n$, it is easy to see that $H_{l}(z)$ satisfies the $b$-th order nonlocal differential equation,

$$
\frac{d^{b} H_{l+1}(z)}{d z^{b}}=2 H_{l}(z / 2) e^{z / 2},
$$

for all $l \geq 1$. This recursion in Eq. (A2) starts from the initial function $H_{1}(z)$ which needs to be computed separately. Using $F_{1}(n)=0$ for $0 \leq n \leq(b-1)$ and $F_{1}(n)=1$ for $n \geq b$, we find $H_{1}(z)=\sum_{k=0}^{b-1} z^{k} / k$ !. The next step is to define a new function,

$$
U_{l}(z)=H_{l}(z) e^{-z}=\sum_{n=0}^{\infty} F_{l}(n) \frac{z^{n}}{n !} e^{-z} .
$$

From Eq. (A1), it follows, after a few steps of algebra, that $U_{l}(z)$ satisfies the differential equation,

$$
\sum_{k=0}^{b}\left(\begin{array}{l}
b \\
k
\end{array}\right) \frac{d^{k} U_{l+1}(z)}{d z^{k}}=2 U_{l}(z / 2),
$$

for all $l \geq 1$ starting with the initial function, $U_{1}(z)=$ $e^{-z} \sum_{k=0}^{b-1} z^{k} / k !$

We now define the Laplace transform, $\tilde{U}_{l}(s)=$ $\int_{0}^{\infty} U_{l}(z) e^{-s z} d z$. Taking the Laplace transform in Eq. (A3), we get

$$
\tilde{U}_{l}(s)=\sum_{n=0}^{\infty} F_{l}(n) \frac{1}{(1+s)^{n+1}}=\tilde{F}_{l}(s)
$$

where we have used the identity $\int_{0}^{\infty} e^{-z} z^{n} d z=n$ ! and the definition of $\tilde{F}_{l}(s)$ in Eq. (5). Next we take the Laplace transform on both sides of Eq. (A4). Using the initial conditions for $n=0$, one can show easily that $d^{k} U_{l}(z) /\left.d z^{k}\right|_{z=0}=0$ for all $l \geq 1$ and $k \leq(b-1)$. Using this condition and doing integration by parts, one finds

$$
\sum_{k=0}^{b}\left(\begin{array}{l}
b \\
k
\end{array}\right) s^{k} \tilde{U}_{l+1}(s)=4 \tilde{U}_{l}(2 s) .
$$

Summing the left hand side of Eq. (A6) and identifying $\tilde{U}_{l}(s)=\tilde{F}_{l}(s)$ as in Eq. (A5) then gives the desired recursion relation

$$
\tilde{F}_{l+1}(s)=\frac{4}{(1+s)^{b}} \tilde{F}_{l}(2 s) .
$$

[1] T.A. Witten and L.M. Sander, Phys. Rev. Lett. 47, 1400 (1981).

[2] R. Brady and R.C. Ball, Nature 309, 225 (1984); L. Niemeyer, L. Pietronero, and H.J. Wiesmann, Phys. Rev. Lett. 52, 1033 (1984); J. Nittmann, G. Daccord, and H.E. Stanley, Nature 314, 141 (1985).

[3] P.G. Saffman, G.I. Taylor, Proc. R. Soc. London, Ser A 245, 2312 (1958); B. Shraiman and D. Bensimon, Phys. Rev. A 30, 2840 (1984).

[4] D. Grier, E. Ben-Jacob, R. Clarke, and L.M. Sander, Phys. Rev. Lett. 56, 1264 (1986).

[5] T. Vicsek, Fractal Growth Phenomena, World Scientific, Singapore, 1989.

[6] For a nice, short review on recent developments, see T.C. Halsey, Phys. Today, 53, 36 (2000).

[7] R.M. Bradley and P.N. Strenski, Phys. Rev. B 31, 4319 (1985).

[8] D. Aldous and P. Shields, Probab. Th. Rel. Fields 79, 509 (1988).

[9] D. E. Knuth, The Art of Computer Programming, Vol. 3, Sorting and Searching, 2nd ed. (Addison-Wesley, Reading, 1998), see page 489; R. Sedgewick, Algorithms, 2nd ed. (Addison-Wesley, Reading, 1988), see page 245.

[10] H. Mahmoud, Evolution of Random Search Trees, (Wiley, New York, 1992).

[11] P. Flajolet and R. Sedgewick, SIAM J. of Comput. 15, 748 (1986).

[12] P. Flajolet and B. Richmond, Random Struc. Algor. 3, 305 (1992).

[13] P. Kirschenhofer, H. Prodinger, and W. Szpankowski, SIAM J. of Comput. 23, 598 (1994).

[14] G. Louchard and W. Szpankowsky, IEEE Trans. Inform. Theory 41, 478 (1995) and references therein.

[15] C. Knessl and W. Szpankowsky, SIAM J. Comput. 30, $923(2000)$.

[16] M.B. Hastings and T.C. Halsey, Europhys. Lett. 55, 679 (2001). 
[17] A. Kolmogorov, I. Petrovsky, and N. Piscounov, Moscou Univ. Bull. Math. A 1, 1 (1937); translated and reprinted in P. Pelce, Dynamics of Curved Fronts (Academic Press, San Diego, 1988).

[18] R. A. Fisher, Ann. of Eugenics 7, 355 (1937).

[19] J. D. Murray, Mathematical Biology (Springer-Verlag, New York, 1989).

[20] For a review see U. Ebert and W. van Saarloos, Physica D 146, 1 (2000).

[21] P. L. Krapivsky and S. N. Majumdar, Phys. Rev. Lett. 85, 5492 (2000); S. N. Majumdar and P. L. Krapivsky, Phys. Rev. E 62, 7735 (2000); D. S. Dean and S. N. Majumdar, Phys. Rev. E 64, 046121 (2001); S.N. Majumdar and P.L. Krapivsky, Phys. Rev. E 63, 045101 (R) (2001); for a recent brief review of these results see S.N. Majumdar and P.L. Krapivsky, Physica A 318, 161 (2003).

[22] S.N. Majumdar and P.L. Krapivsky, Phys. Rev. E 65, 036127 (2001).

[23] E. Ben-Naim, P.L. Krapivsky and S.N. Majumdar, Phys. Rev. E 64, 035101 (R) (2001).

[24] P.L. Krapivsky and E. Ben-Naim, cond-mat/0302525.

[25] J. Ziv and A. Lempel, IEEE Trans. Information Theory 23, 337 (1977); J. Ziv, IEEE Trans. Inform. Theory 24, 405 (1978);

[26] B. Pittel, Ann. Probab. 13, 414 (1985); Adv. Appl. Probab. 18, 139 (1986). 